\title{
Output Consistency Scale to Standardize Ostomate Output Description in Clinical Practice and Studies
}

\author{
Lisa A Whisenhunt ${ }^{1}$, Linda $\mathrm{H} \mathrm{Xu}^{2}$, Fan Yang ${ }^{2}$ and Jacques Izard ${ }^{1,2,3 *}$ \\ ${ }^{1}$ Nebraska Food for Health Center, University of Nebraska-Lincoln, USA \\ ${ }^{2}$ Department of Food Science and Technology, University of Nebraska-Lincoln, USA \\ ${ }^{3}$ Fred and Pamela Buffet Cancer Center, University of Nebraska Medical Center, Omaha, USA
}

*Corresponding author: Jacques Izard, Department of Food Science and Technology, University of Nebraska-Lincoln, Lincoln, USA.

Received Date: September 17, 2021

Published Date: October 07, 2021

\section{Introduction}

Stool descriptors have become popular due to the large diffusion of the Bristol Stool Form Scale (BSFS) via clinical studies, clinical trials, and social media. The applications have been numerous and centered around standardization of terminology that can be used by health care professionals and patients alike, as well as individuals interested in their wellness and the associated partners in the wellness industry. For a portion of the population, the digestive content is rerouted to an external manufactured pouch or bag, making the use of the BSFS visual descriptors of stool difficult. From day one post-resection surgery, ostomates are challenged with output management. The lack of standardized descriptors may hinder proper communication between the individual and the support team, as well as providing proper characterization in clinical studies and clinical trials.

We propose the Lincoln Ostomy Output Consistency Scale for jejunostomy, ileostomy and colostomy (LOOCS) to overcome the limitations of the BSFS for qualifying ostomy outputs. The design was based on the need to describe perceived consistency from the ostomate point of view. We anticipate that the LOOCS scale can be effective in pediatric and adult clinical research settings, as well as self-monitoring to manage the quality of life.

Keywords: Bristol Stool Form Scale; Ostomy; Output; Stoma; Defecation; Gastrointestinal disorders; Precision nutrition

\section{Introduction}

When the original Bristol Stool Form Scale was published, the authors wrote that "of all bodily functions defecation is perhaps the least understood and least studied" [1]. Since then, thousands of publications have addressed this issue directly or indirectly. The direct benefit is the standardization of stool descriptors, enabling both patients and health care professionals to use the same language. Additionally, this scale avoids having the individual to provide a stool for water content analysis, or stool rheology and viscosity measurements that are not practical for the patient or participant in clinical studies. Many applications have derived from that standardization, such as associating stool form and digestive transit time [2], linking the microbiome characteristics to the scale scoring [3], incorporating the evaluation in clinical trials and clinical studies to understand the impact of a treatment [4] or a diet [5], improving medical stool testing [6], etc.

For a portion of the population, the digestive content is rerouted to an external collection system on the abdomen. Ostomates have available a variety of manufactured pouches or bags with different characteristics to enable an active life within the community. Colon cancer, cancer of the cervix or endometrium, Crohn's disease or ulcerative colitis escalation, and traumatic abdominal injury can lead to a stoma forming surgery. For individuals with a permanent 
ostomy or awaiting an ostomy reversal, from day one, they are challenged with food intake and output management. Diet and fluid intake are tightly linked to aspects of stoma output such as consistency, volume, and frequency. To date, there is no standardized output scale available for this population with very different stool characteristics compared to individuals with unaltered digestive tract.

The stool form scales have evolved over the years to respond to the needs of different populations with an oral-anal digestive continuum. The modifications encompass the addition or removal of choices [1,2, 7] and modifications for pediatric use [8]. Visual scales supported by written description have been shown to require no training for the patients, physicians, and nurses to remain effective even after translation in other languages [9]. A new scale has been designed to answer the specific needs for nontoilet-trained children and referred to as the Brussels Infants and Toddlers Stool Scale [10]. We are proposing the Lincoln Ostomy Output Consistency Scale (LOOCS) for jejunostomy, ileostomy and colostomy to enable standardization of output description.

\section{Discussion}

The limitations of the Bristol Stool Form Scale (BSFS) were underlined in one of our recent studies including ileostomates, colostomates and individuals with unaltered digestive tract, traditionally mentioned as healthy controls. The BSFS Types, while descriptive, do not really reflect the characteristics of the stool stored in the ostomy bag, thus providing limited information for comparison. The clinical study was approved by the University of Nebraska-Lincoln Institutional Review Board (IRB Approval \#20190819166EP; Human Subject Federalwide Assurance Number FWA00002258). Participants provided consent. The recruited individuals, over the age of 19 , included ileostomates $(n=24)$, colostomates $(\mathrm{n}=21)$ and individuals with unaltered digestive tract $(n=23)$. The design was a cross-sectional sample collection in which participants provided a descriptor of their stool/output using the Bristol Stool Form Scale $(n=68)$. Indicating one or two BSFS types was authorized.

The ileostomates population reported a range from Type 6 to 7 , while colostomates reported a range from Type $3 / 4$ to 7 . The control population with an unaltered digestive tract, reported a range from Type 1 to 6/7. More precisely, for ileostomates, the answers were as follows: Type 6 for 7 individuals, Type 6/7 for 5 , and Type 7 for 12 . For colostomates, the answers were as follows: Type 3/4 for 1 individual, Type 4 for 10, Type 5 for 3, Type 6 for 6 , Type $6 / 7$ for 1 , and Type 7 for 6 . As expected, the control group used a broader range of the BSFS scale: Type 1 for 4 individuals, Type $1 / 2$ for 1 , Type 2 for 1 , Type 3 for 2 , Type $3 / 4$ for 1 , Type 4 for 10 , Type 5 for 1 , Type 6 for 2 , Type $6 / 7$ for 1 .

We noted that the BSFS types, for ostomates, is a compressed scale that does not fully compare to their experience regarding in particular higher water digestive content. The peculiar aspect of having the stool/output going to a manufactured pouch alters the visual description classically used in the BSFS and the participant has to rely more on consistency than visual stool descriptors. The design of the Lincoln Ostomy Output Consistency Scale for jejunostomy, ileostomy and colostomy (LOOCS) were based on the need to describe the perceived consistency of the output from the ostomate's point of view. We combined our experience of clinical studies, the availability of data regarding output, and the need to overlap with BSFS while expending the standardization of stool/ output description. The design was performed with feedback from the ostomy community. The nine Types use letters (A through I) to avoid confusion with BSFS and derivations $[1,2,7,8]$. Type A ("Separated formed output") in LOOCS is comparable to Type 1 ("Separate hard lumps"') of the BSFS. LOOCS Type C ("Semi-formed output") is comparable to BSFS Type 4 ("Like a sausage or snake, smooth and soft"). The remaining types in the LOOCS encompass the Type 5 to 7 of the BSFS. Three formed outputs have been included (Types A through C): separated formed output, firm formed output, and semi-formed output. Three paste consistencies have been included (Types D through F): semi-solid, soft-solid, and pourable paste. Three liquid consistencies have been included (Types G through I): creamy, thin opaque, and clear liquid. Two versions of the scale were created, one as a quick reference guide (Table 1), and the other one designed for the visual learners and individuals requiring examples of similar consistencies (Figure 1). In Figure 1, the Type descriptor was complemented both with an example of matching consistency and an image related to that example.

Table 1: Short format Lincoln Ostomy Output Consistency Scale for jejunostomy, ileostomy and colostomy (sfLOOCS).

\begin{tabular}{|c|c|}
\hline Type A & Separated formed output \\
\hline Type B & Firm formed ouput \\
\hline Type C & Semi-formed output \\
\hline Type D & Semi-solid paste \\
\hline Type E & Soft-solid paste \\
\hline Type F & Pourable paste \\
\hline Type G & Creamy liquid \\
\hline Type H & Thin opaque liquid \\
\hline Type I & Clear liquid \\
\hline
\end{tabular}




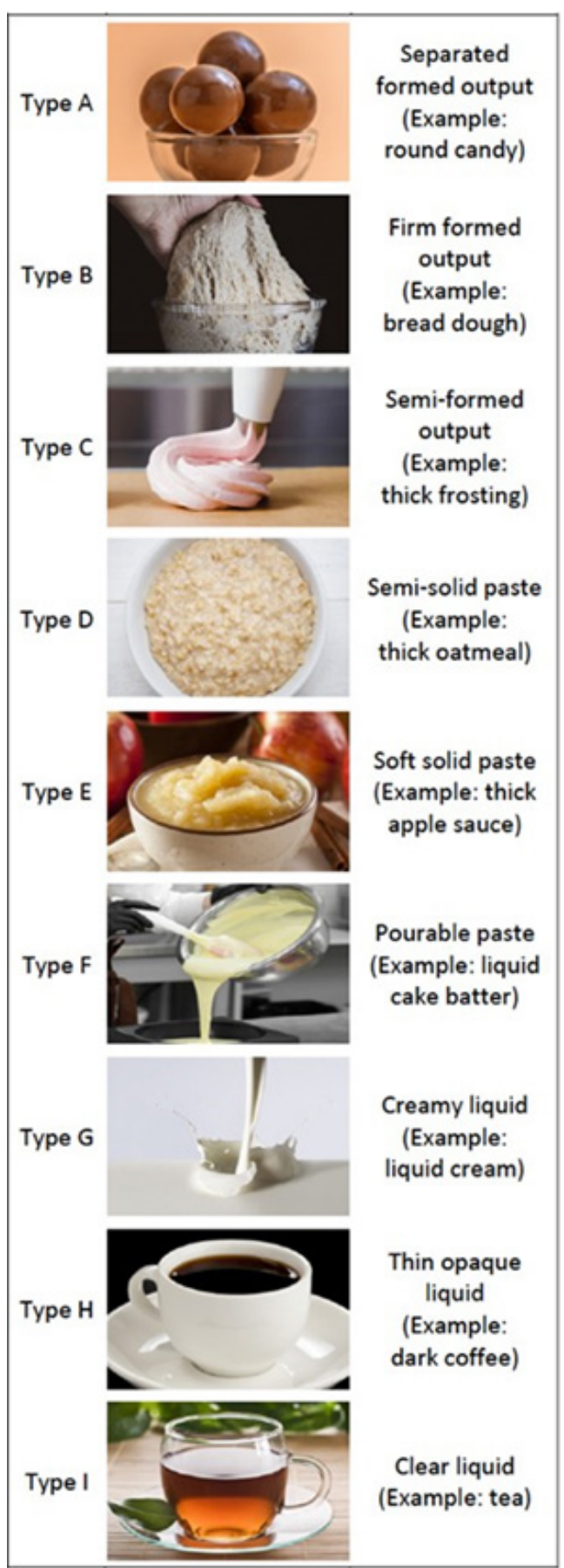

Figure 1: Lincoln Ostomy Output Consistency Scale for jejunostomy, ileostomy and colostomy (LOOCS).

Stool appearance and consistency are important semiologic parts of the clinical management of digestive output in ostomates. A patient's description of his, her, or their output that can be matched with standardized descriptors by health care professionals is important. Both the individuals and the health care team should have the same language in describing the day-to-day changes that might be linked to a specific diagnostic, changes in medication, or diet modification. Outside of the clinical setting, ostomates, by tracking their output, should be able to monitor and manage their health problems. Thus, taking precautionary measures based on past experience in the context of the standardized evaluations, and enhancing their participation in precision nutrition. The LOOCS should help healthcare professionals in both traditional and emergency room settings, as well as benefit clinical studies and trials by providing a standardization across studies.

\section{Acknowledgement}

We thank the participants in the clinical study, and the laboratory personnel who helped, as well as the ostomate 
community members who provided comments on the LOOCS. The imagery was acquired from Shutterstock, Inc. (New York City, NY, USA). Study data were collected and managed using REDCap electronic data capture tools hosted at the University of NebraskaLincoln. This work was supported by a grant from the Nebraska Food for Health Center of the University of Nebraska-Lincoln to JI and by the Hatch Multistate Research capacity funding program W4122 from the USDA National Institute of Food and Agriculture.

Clinical study design: JI, LAW

Methodology and data collection: JI, LAW

Data analysis: JI, LAW

Scale design: JI, FY, LAW, LHX

Literature research: JI

Writing manuscript: JI

Editing manuscript: JI, FY, LAW, LHX

\section{Conflict of Interest}

No conflict of interest.

\section{References}

1. KW Heaton, J Radvan, H Cripps, RA Mountford, FE Braddon, et al. (1992) Defecation frequency and timing, and stool form in the general population: a prospective study. Gut 33(6): 818-824.

2. Lewis SJ, KW Heaton, (1997) Stool form scale as a useful guide to intestinal transit time. Scand J Gastroenterol 32(9): 920-924.
3. Doris Vandeputte, Gwen Falony, Sara Vieira-Silva, Raul Y Tito, Marie Joossens, et al. (2016) Stool consistency is strongly associated with gut microbiota richness and composition, enterotypes and bacterial growth rates. Gut 65(1): 57-62.

4. QiQi Zhou, Meghan L Verne, Jeremy Z Fields, John J Lefante, Sarpreet Basra, et al. (2019) Randomised placebo-controlled trial of dietary glutamine supplements for postinfectious irritable bowel syndrome. Gut 68(6): 996-1002.

5. Zenta Yasukawa, Ryo Inoue, Makoto Ozeki, Tsutomu Okubo, Tomohisa Takagi, et al. (2019) Effect of Repeated Consumption of Partially Hydrolyzed Guar Gum on Fecal Characteristics and Gut Microbiota: A Randomized, Double-Blind, Placebo-Controlled, and Parallel-Group Clinical Trial. Nutrients 11(9): 2170.

6. Caroff DA, PH Edelstein, K Hamilton, DA Pegues (2014) The Bristol stool scale and its relationship to Clostridium difficile infection. I Clin Microbiol 52(9): 3437-3439.

7. GJ Davies, M Crowder, B Reid, JW Dickerson (1986) Bowel function measurements of individuals with different eating patterns. Gut 27(2): 164169.

8. Mariella M Lane, Danita I Czyzewski, Bruno P Chumpitazi, Robert J Shulman (2011) Reliability and validity of a modified Bristol Stool Form Scale for children. J Pediatr 159(3): 437-441.

9. D Parés, M Comas, D Dorcaratto, MI Araujo, M Vial, et al. (2009) Adaptation and validation of the Bristol scale stool form translated into the Spanish language among health professionals and patients. Rev Esp Enferm Dig 101(5): 312-316.

10. Koen Huysentruyt, Ilan Koppen, Marc Benninga, Tom Cattaert, Jiqiu Cheng, et al. (2019) The Brussels Infant and Toddler Stool Scale: A Study on Interobserver Reliability. J Pediatr Gastroenterol Nutr 68(2): 207213. 